\title{
Modulation of pure spin currents with a ferromagnetic insulator
}

\author{
Estitxu Villamor, ${ }^{1}$ Miren Isasa, ${ }^{1}$ Saül Vélez, ${ }^{1}$ Amilcar Bedoya-Pinto, ${ }^{1}$ Paolo Vavassori,,${ }^{1,2}$ Luis E. Hueso, ${ }^{1,2}$ \\ F. Sebastián Bergeret, ${ }^{3,4}$ and Fèlix Casanova ${ }^{1,2, *}$ \\ ${ }^{1}$ CIC nanoGUNE, 20018 Donostia-San Sebastian, Basque Country, Spain \\ ${ }^{2}$ IKERBASQUE, Basque Foundation for Science, 48011 Bilbao, Basque Country, Spain \\ ${ }^{3}$ Centro de Física de Materiales (CFM-MPC) Centro Mixto CSIC-UPV/EHU, 20018 Donostia-San Sebastian, Basque Country, Spain \\ ${ }^{4}$ Donostia International Physics Center (DIPC), 20018 Donostia-San Sebastian, Basque Country, Spain \\ (Received 8 April 2014; revised manuscript received 20 September 2014; published 9 January 2015)
}

\begin{abstract}
We propose and demonstrate spin manipulation by magnetically controlled modulation of pure spin currents in cobalt/copper lateral spin valves, fabricated on top of the magnetic insulator $\mathrm{Y}_{3} \mathrm{Fe}_{5} \mathrm{O}_{12}$ (YIG). The direction of the YIG magnetization can be controlled by a small magnetic field. We observe a clear modulation of the nonlocal resistance as a function of the orientation of the YIG magnetization with respect to the polarization of the spin current. Such a modulation can only be explained by assuming a finite spin-mixing conductance at the $\mathrm{Cu} / \mathrm{YIG}$ interface, as it follows from the solution of the spin-diffusion equation. These results open a path towards the development of spin logics.
\end{abstract}

DOI: 10.1103/PhysRevB.91.020403

PACS number(s): 72.25.Ba, 72.25.Mk, 75.47.Lx

Spintronics is a rapidly growing field that aims at using and manipulating not only the charge, but also the spin of the electron, which could lead to faster data processing, nonvolatility, and lower electrical power consumption as compared to conventional electronics [1]. Sophisticated applications such as hard-disk read heads and magnetic random access memory (MRAM) have been introduced in the last two decades.

Further progress could be achieved with pure spin currents, which are an essential ingredient in an envisioned spin-only circuit that would integrate logics and memory [2]. The most basic unit in such a concept is the spin analog to the transistor, in which the manipulation of pure spin currents is crucial. The original proposal by Datta and Das [3], which is also applicable to pure spin currents [4], suggested a spin manipulation that would arise from the spin precession due to the spin-orbit interaction modulated by an electric field (Rashba coupling). However, a fundamental limitation appears here, because the best materials for spin transport are those showing the lowest spin-orbit interaction and, therefore, there has been no success in electrically manipulating the spins and propagating them at the same environment, with few exceptions [4].

Alternative ways to control pure spin currents are thus desirable. One could take advantage of the spin-mixing conductance concept [5,6] at nonmagnetic metal (NM)/ferromagnetic insulator (FMI) interfaces, which governs the interaction between the spin currents present at the NM and the magnetization of the FMI. This concept is the basis of new spindependent phenomena, including spin pumping [6-12], spin Seebeck effect $[6,13]$, and spin Hall magnetoresistance (SMR) [6,14-18]. In these cases, a NM with large spin-orbit coupling is required to convert the involved spin currents into charge currents via the inverse spin Hall effect [19].

In this Rapid Communication, we demonstrate an alternative way of modulating pure spin currents based on a magnetic, instead of electric, gating. To that end, we use lateral spin valves (LSVs). These devices allow an electrical injection and detection of pure spin currents in a NM channel by using

*f.casanova@nanogune.eu ferromagnetic (FM) electrodes in a nonlocal configuration [20-29]. The LSVs have been fabricated on top of a FMI, in order to enable the magnetic gating of the pure spin currents. The basic idea is depicted in Fig. 1: When the spin polarization $(s)$ has the same direction as the magnetization $(M)$ of the FMI, the spin current reaching the detector will not vary with respect to the case where no FMI is used [Fig. 1(a)]. However, when $s$ and $M$ are noncollinear, part of the spin current will be absorbed by $M$ via a spin-transfer torque [30-32], leading to maximum spin absorption for perpendicular $M$ and $s$ [Fig. 1(b)]. By using LSVs, we are able to extract the spin-mixing conductance of NM/FMI interfaces in the absence of charge currents, which otherwise could lead to spurious effects, as suggested by some authors [33,34]. Furthermore, the use of NM metals with a low atomic number, employed in LSVs, rules out spin-orbit interaction effects that might exist for other systems, such as Pt/YIG [35].

We chose $\mathrm{Y}_{3} \mathrm{Fe}_{5} \mathrm{O}_{12}$ (YIG) [36] as a magnetic gate because it is ferromagnetically soft and has a negligible magnetic anisotropy. $M$ as a function of the applied in-plane magnetic field $(H)$ measured by a vibrating sample magnetometer (VSM) saturates at $\sim 100$ Oe [Fig. 2(a)], allowing control of $M$ above this field. Cobalt (Co)/copper (Cu) LSVs were fabricated on top of YIG by two-step electron-beam lithography, ultrahigh-vacuum evaporation, and a lift-off process [Fig. 2(b)] [37]. Ar-ion milling was performed prior to the $\mathrm{Cu}$ deposition in order to remove resist leftovers [37]. To overcome the low spin injection of Co when using transparent interfaces [21-23], an oxide layer was created at the $\mathrm{Co} / \mathrm{Cu}$ interface by letting $\mathrm{Co}$ oxidize after milling and before $\mathrm{Cu}$ deposition. The presence of an interface resistance, estimated to be $R_{I} \geqslant 5 \Omega$, is known to enhance the spin injection efficiency $[24,25]$. The LSVs were bridged by the same $\mathrm{Cu}$ channel, with thickness $t \sim 100 \mathrm{~nm}$, width $w \sim 200 \mathrm{~nm}$, and different edge-to-edge distances $(L)$ between the FM electrodes [37].

All measurements were performed using a "dc reversal" technique [27] in a liquid-He cryostat with an applied magnetic field $H$ at a temperature of $150 \mathrm{~K}$. The sample can be rotated in plane in order to change the direction of $H$, which is given by the angle $\alpha$ defined in Fig. 2(b). The nonlocal voltage $V_{\mathrm{NL}}$ 

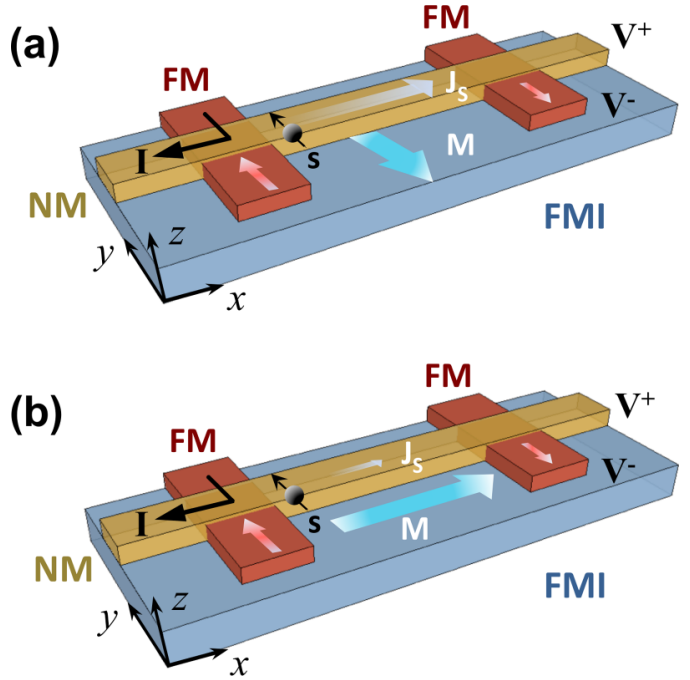

FIG. 1. (Color online) Scheme of the device used to modulate a pure spin current with magnetic gating. It consists of a ferromagnetic (FM)/nonmagnetic (NM) lateral spin valve on top of a ferromagnetic insulator (FMI). The nonlocal measurement configuration is shown. The $x, y$, and $z$ axes are indicated as used in the text. (a) When the magnetization of the FMI $(M)$ and the polarization $(s)$ of the injected pure spin current $\left(j_{s}\right)$ are parallel, there will be no spin absorption. (b) When $M$ and $s$ are perpendicular, the spin absorption will be maximum.

measured at the detector, normalized to the injected current $I$, is defined as the nonlocal resistance $R_{\mathrm{NL}}=V_{\mathrm{NL}} / I$ [Fig. 2(b) shows a measurement scheme]. First, in order to check the standard performance of the LSV, the direction of $H$ was fixed parallel to the FM electrodes $\left(\alpha=0^{\circ}\right)$ and its value was swept from positive to negative, and vice versa, while $R_{\mathrm{NL}}$ was measured. This is plotted in Fig. 2(c), where $R_{\mathrm{NL}}$ changes from positive to negative when the relative magnetization of the FM electrodes changes from parallel (P) to antiparallel (AP) by sweeping $H$. This measurement is an unambiguous demonstration that a pure spin current is transported along the $\mathrm{Cu}$ channel [20-29]. It is worth noting that the relative magnetization of the Co electrodes changes at $H \geqslant 400 \mathrm{Oe}$, far above the saturation field of YIG $(\sim 100$ Oe). This detail is important for the performance of the next measurement, which consists in measuring $R_{\mathrm{NL}}$ while fixing the value of $H$ and sweeping $\alpha$. As shown in Fig. 2(d), this was done for both the $\mathrm{P}$ and $\mathrm{AP}$ configurations of the Co electrodes, which can be chosen with the proper magnetic field history. In this case, $H$ was fixed to 250 Oe [see the dots in Fig. 2(c)], which is large enough to control $M$ of YIG but not to rotate the magnetization of the Co electrodes, as confirmed by magnetooptical Kerr effect (MOKE) microscopy [37,38]. As intended, Fig. 2(d) shows a clear modulation of the measured $R_{\mathrm{NL}}$ (i.e., a modulation of the spin current) when $M$ of YIG is rotated in plane, clearly demonstrating a direct magnetic gating to a pure spin current. The reflection symmetry between the $\mathrm{P}$ and AP modulations again rules out the possibility of a relative tilting between the magnetization of Co electrodes [39]. In addition, the measurements were repeated in a control sample, fabricated on a $\mathrm{SiO}_{2}$ substrate, in order to exclude any other possible artifacts [37].

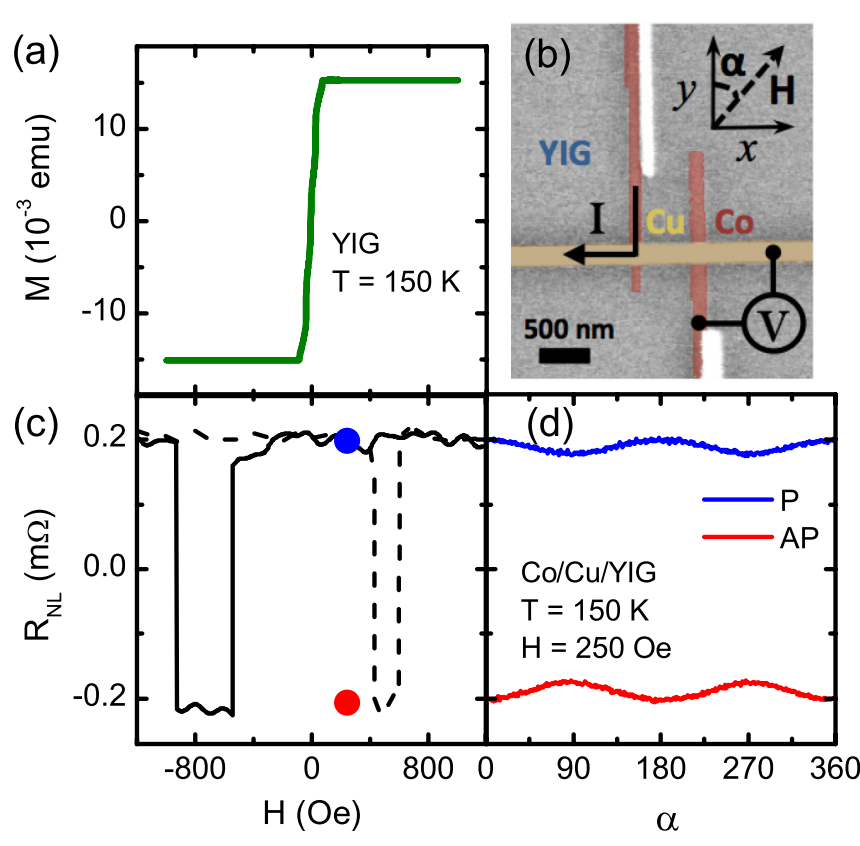

FIG. 2. (Color online) (a) Magnetization of YIG $(M)$ as a function of the applied in-plane magnetic field $H$ measured at $150 \mathrm{~K}$. (b) Colored scanning electron microscopy (SEM) image of a LSV. The nonlocal measurement configuration, materials, direction of $H$, and its angle $\alpha$ with respect to the FM electrodes are shown. (c) Nonlocal resistance $\left(R_{\mathrm{NL}}\right)$ measured at $150 \mathrm{~K}$ as a function of $H$ with $\alpha=0^{\circ}$ for a LSV with a separation distance between Co electrodes of $L=1.6 \mu \mathrm{m}$. The solid (dashed) line indicates the decreasing (increasing) sweep of $H$. A constant background of $0.14 \mathrm{~m} \Omega$ is subtracted from the data. Blue and red dots correspond to the value of $R_{\mathrm{NL}}$ at the parallel (P) and antiparallel (AP) configurations of the Co electrodes, respectively, at $H=250 \mathrm{Oe}$. (d) $R_{\mathrm{NL}}$ as a function of $\alpha$, measured for both the $\mathrm{P}$ and $\mathrm{AP}$ configurations, at $150 \mathrm{~K}$ with $H=250$ Oe for the same LSV.

The total change in $R_{\mathrm{NL}}$, caused by the spin absorption at the $\mathrm{Cu} / \mathrm{YIG}$ interface, is defined as the nonlocal modulation $\delta R_{\mathrm{NL}}=R_{\mathrm{NL}}\left(\alpha=0^{\circ}\right)-R_{\mathrm{NL}}\left(\alpha=90^{\circ}\right)$ (tagged in Fig. 3). This figure contains the same data from Fig. 2(d), although, for the sake of clarity, $\mathrm{P}$ and AP configurations are plotted separately. In this case, for an $L$ of $1.6 \mu \mathrm{m}, \delta R_{\mathrm{NL}}$ has a magnitude of $\sim 0.025 \mathrm{~m} \Omega$. We can define the factor $\beta=\delta R_{\mathrm{NL}} / R_{\mathrm{NL}}\left(\alpha=0^{\circ}\right)$ as an analog of a magnetoresistance, which gives a measure of the efficiency of the magnetic gating. Here, $\beta=8.33 \%$ is obtained for the LSV with $L=1.6 \mu \mathrm{m}$, whereas $\beta=2.96 \%$ for $L=570 \mathrm{~nm}$, showing that longer channels provide more efficient modulations.

In order to quantify the observed modulation of $R_{\mathrm{NL}}$, we solve the spin-diffusion equation $[20,21,24]$ in the NM channel,

$$
\nabla^{2} \vec{\mu}_{s}=\frac{\vec{\mu}_{s}}{\lambda^{2}}+\frac{1}{\lambda_{m}^{2}} \vec{\mu}_{s} \times \hat{n},
$$

where $\vec{\mu}_{s}$ is the spin accumulation at the NM, and the vector refers to the spin-polarization direction. $\lambda$ is the spin-diffusion length of the NM and $\lambda_{m}=\sqrt{\frac{D \hbar}{2 \mu_{B}|B|}}$ is the magnetic length determined by the amplitude of the magnetic field $B \hat{n}(\hat{n}$ is the unit vector giving its direction). The last term in Eq. (1) 


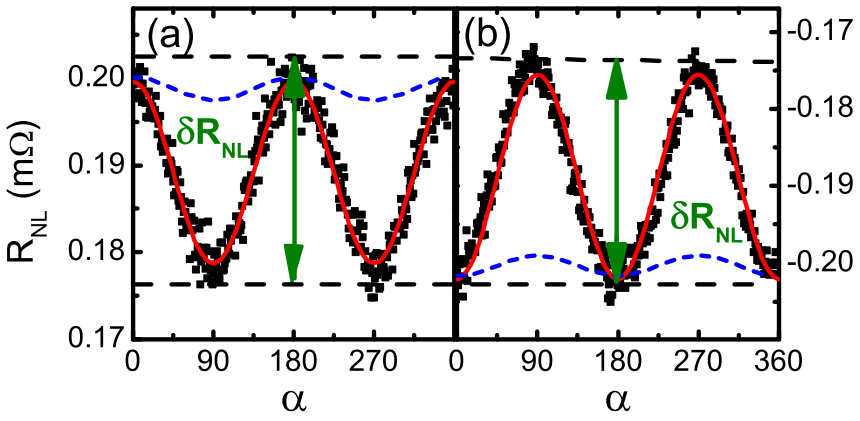

FIG. 3. (Color online) Nonlocal resistance (black solid squares) as a function of the angle $\alpha$ between the FM electrodes and the applied magnetic field $H$, measured for the (a) parallel and (b) antiparallel configurations, at $150 \mathrm{~K}$ and $H=250 \mathrm{Oe}$ for a LSV with a separation distance of $L=1.6 \mu \mathrm{m}$. The red solid line corresponds to the fit of the data to Eq. (2). The blue dashed line corresponds to Eq. (2) in the absence of the spin-mixing conductance of the FMI/NM interface. The nonlocal modulation $\delta R_{\mathrm{NL}}$ is tagged.

describes the well-known spin precession due to the applied field [40,41]. $B$ is proportional to $H$ and, for $\mathrm{Cu}$, we can approximate $B \sim \mu_{0} H$. $D$ is the electronic diffusion constant of the NM, and $\mu_{B}$ is the Bohr magneton. Assuming $t \ll \lambda$, we can integrate Eq. (1) in the $z$ direction and use the BrataasNazarov-Bauer boundary condition at the NM/FMI interface [5]. From the solution one can obtain an expression for the nonlocal resistance at the FM detector that reads [37,42,43]

$$
R_{\mathrm{NL}}=\frac{P_{I}^{2} R_{N}}{2}\left[\cos ^{2} \alpha e^{-L / \lambda}+\sin ^{2} \alpha \operatorname{Re}\left(\frac{\lambda_{1}}{\lambda} e^{-L / \lambda_{1}}\right)\right],
$$

which is only valid in the high interface resistance limit, i.e., if $R_{I} \gg R_{N} . P_{I}$ is the spin polarization of the FM/NM interface at both the FM injector and detector, $R_{N}=\rho \lambda / w t$ is the spin resistance of the NM, and $\rho$ is its electrical resistivity. The length $\lambda_{1}$ is defined as

$$
\lambda_{1}=\frac{\lambda}{\sqrt{1+\frac{2 \rho G_{r} \lambda^{2}}{t}+i\left(\frac{\lambda}{\lambda_{m}}\right)^{2}}},
$$

where $G_{r}$ is the real part of the spin-mixing conductance per unit area [5] of the FMI/NM interface. We have disregarded the imaginary part of the spin-mixing conductance in accordance with Refs. [14,32]. Notice that for $\alpha=0^{\circ}$, the $R_{\mathrm{NL}}$ for the case without FMI $[24,28,29]$ is recovered: $R_{\mathrm{NL}}=\frac{P_{I}^{2} R_{N}}{2} e^{-L / \lambda}$. At $\alpha=90^{\circ}$ we obtain a similar expression for $R_{\mathrm{NL}}$ as in the $\alpha=0^{\circ}$ case, but with a reduced spin-diffusion length $\operatorname{Re}\left(\lambda_{1}\right): R_{\mathrm{NL}}=\frac{P_{I}^{2} R_{N}}{2} \operatorname{Re}\left(\frac{\lambda_{1}}{\lambda} e^{-L / \lambda_{1}}\right)$. Equation (3) shows that two quantities renormalize the spin-diffusion length: the spinmixing conductance by means of the real term $2 \rho G_{r} \lambda^{2} / t$, and the imaginary Hanle term $i\left(\lambda / \lambda_{m}\right)^{2}$ originating from the applied field. While the former leads to a reduction of $\lambda$ due to the torque exerted by the NM/FMI interface to the spins $[30,32]$, the latter causes, in addition, the precession of the spins when the $s$ and $H$ are noncollinear [40].

At first glance, one might think that the Hanle term could be enough to explain the observed modulation of $R_{\mathrm{NL}}$ as a function of $\alpha$. However, as shown in Fig. 3, a field of 250 Oe in the absence of $G_{r}$ leads to a modulation of $R_{\mathrm{NL}}$

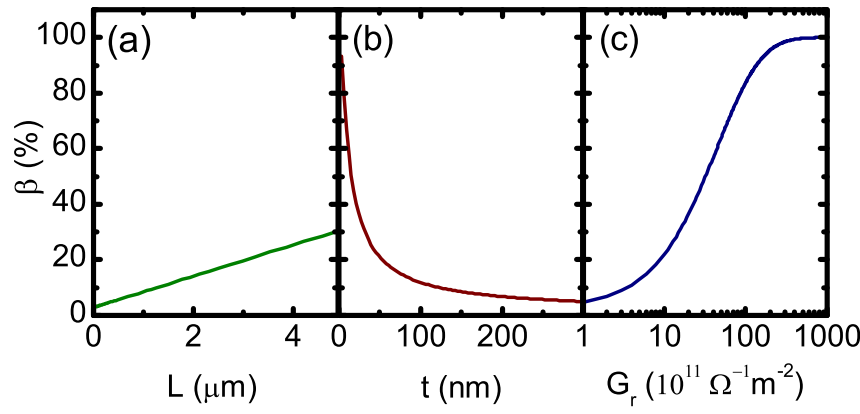

FIG. 4. (Color online) Representation (solid lines) of the $\beta$ factor, based on Eq. (2) for an applied magnetic field $H=250 \mathrm{Oe}$, as a function of (a) the distance $(L)$ between FM electrodes, (b) the thickness $(t)$ of the NM channel, and (c) the spin-mixing conductance per unit area $\left(G_{r}\right)$ of the NM/FMI interface. The parameters used for the simulation are as follows: (a) $\lambda=522 \mathrm{~nm}$, $\rho=2.1 \mu \Omega \mathrm{cm}, G_{r}=5 \times 10^{11} \Omega^{-1} \mathrm{~m}^{-2}$, and $t=100 \mathrm{~nm}$. (b) $\lambda=$ $522 \mathrm{~nm}, \rho=2.1 \mu \Omega \mathrm{cm}, G_{r}=5 \times 10^{11} \Omega^{-1} \mathrm{~m}^{-2}$, and $L=1.6 \mu \mathrm{m}$. (c) $\lambda=522 \mathrm{~nm}, \rho=2.1 \mu \Omega \mathrm{cm}, L=1.6 \mu \mathrm{m}$, and $t=100 \mathrm{~nm}$.

(blue dashed line) which is one order of magnitude smaller than the measured one. This is experimentally confirmed in the control sample performed on top of $\mathrm{SiO}_{2}$ [37]. Increasing $H$ would eventually lead to a Hanle effect of the same order as the $G_{r}$ effect. Nevertheless, our experiment is limited to low magnetic fields $(H<400 \mathrm{Oe})$, to avoid the magnetization of the Co electrodes being affected by the direction of $H$, and thus the Hanle term will not be dominant.

Considering both the $G_{r}$ and Hanle terms, Eq. (2) accurately fits the measured $R_{\mathrm{NL}}$ (Fig. 3), reproducing the observed modulation of the spin current. Note also that Eq. (2) reproduces the reflection symmetry between the $\mathrm{P}$ and AP configurations, because the product $P_{I}^{2}$ has an opposite sign for each configuration. The fact that the modulation is observed in a pure spin current in a metal such as $\mathrm{Cu}$ excludes any proximity effect as the origin of the modulation [33,34], confirming the validity of the $G_{r}$ concept.

There are two fitting parameters, $P_{I}$ and $G_{r}$, whereas $w, t$, and $L$ are known geometrical parameters, and $\rho(2.1 \mu \Omega \mathrm{cm})$ and $\lambda(522 \mathrm{~nm})$ are obtained from resistance measurements and $R_{\mathrm{NL}}$ measurements as a function of $L$ [37].

From the fitting for the LSV with $L=1.6 \mu \mathrm{m}$ (Fig. 3), we obtained $P_{I}=0.128 \pm 0.001$ and $G_{r}=(4.28 \pm 0.06) \times$ $10^{11} \Omega^{-1} \mathrm{~m}^{-2}$ for the P state [Fig. 3(a)], and $P_{I}=0.129 \pm$ 0.001 and $G_{r}=(5.63 \pm 0.07) \times 10^{11} \Omega^{-1} \mathrm{~m}^{-2}$ for the AP state [Fig. 3(b)], which are almost identical for both magnetic configurations. Therefore, the value of $G_{r}$ obtained for this particular $L$ is $(4.96 \pm 0.68) \times 10^{11} \Omega^{-1} \mathrm{~m}^{-2}$. The same fitting was performed for the LSV with $L=570 \mathrm{~nm}$, where it was also possible to measure $R_{\mathrm{NL}}$ as a function of $\alpha$, obtaining $P_{I}=0.123 \pm 0.001$ and $G_{r}=(2.82 \pm 0.66) \times$ $10^{11} \Omega^{-1} \mathrm{~m}^{-2}$. Since $G_{r}$ is extracted separately for each device, this transfers the unavoidable device-to-device dispersion (spin transport is very sensitive to any minor defect) into the value of $G_{r}$. The difference, which is less than a factor of 2 , can thus be considered to be small, taking into account that, in order to observe a relevant variation in $\beta$, a much larger change in $G_{r}$ is needed [Fig. 4(c)]. Whereas $P_{I}$ is within the range reported in similar systems [22,28,29], $G_{r}$ 
is substantially smaller than the values obtained for Pt/YIG (ranging from $1.2 \times 10^{12}$ to $6.2 \times 10^{14} \Omega^{-1} \mathrm{~m}^{-2}$ ) [6-9,14-16], $\mathrm{Ta} / \mathrm{YIG}\left(4.3 \times 10^{13} \Omega^{-1} \mathrm{~m}^{-2}\right)$ [16], and Au/YIG (between $3.5 \times 10^{13}$ and $1.9 \times 10^{14} \Omega^{-1} \mathrm{~m}^{-2}$ ) [10,11] either by SMR or spin pumping experiments.

There is a possibility of underestimating $G_{r}$ if the assumption for Eq. (2), $R_{I} \gg R_{N}$, is not fulfilled. For $\beta \sim 8 \%, G_{r}$ would increase by a factor of $\sim 2$, to $\sim 8 \times 10^{11} \Omega^{-1} \mathrm{~m}^{-2}$, by considering transparent interfaces [37], which is still low compared to other NM/YIG interfaces. Another possible reason for the low $G_{r}$ value could be the Ar-ion milling performed before the $\mathrm{Cu}$ deposition [12] or the YIG surface quality. We rule this out by performing a control experiment in Pt/YIG where we obtain $G_{r}=3.34 \times 10^{13} \Omega^{-1} \mathrm{~m}^{-2}$ from SMR measurements $[37,44,45]$. Particularities of the grain structure and the growth condition of the evaporated $\mathrm{Cu}$ on YIG could also lead to an effective reduction of $G_{r}$ at the interface. Alternatively, the spin-orbit interaction effects that might exist for Pt/YIG, Au/YIG, or Ta/YIG [35] could lead to an overestimation of the obtained $G_{r}$ in these systems. Such effects are unlikely in $\mathrm{Cu} / \mathrm{YIG}$. It is worth noting that the $G_{r}$ of a NM/YIG interface, for a NM with a negligible spin-orbit coupling, was not experimentally measured before due to the need of the inverse spin Hall effect (and thus a high spin-orbit coupling metal) in the experiments made so far [6-16].

Finally, a representation of $\beta$, based on Eq. (2), is plotted in Fig. 4 as a function of different parameters $\left(L, t\right.$, and $\left.G_{r}\right)$ which can be controlled in order to improve the efficiency of the magnetic gating. The values of the different parameters used for the representation are listed in the caption and correspond to realistic values taken from our devices. $\beta$ increases linearly with the length $(L)$ between the FM electrodes, reaching $~ 30 \%$ for $L=5 \mu \mathrm{m}$ [Fig. 4(a)]. When the spin current flows over a longer distance, the spin scattering and absorption caused by the NM/FMI interface will be enhanced (i.e., $\beta$ will be larger). This is in agreement with our experimental results discussed above. However, there is an experimental limit, since the nonlocal signal decays exponentially and will be negligible when $L \gg \lambda[23,26]$. By decreasing the thickness $(t)$ of the $\mathrm{Cu}$ channel, $\beta$ increases asymptotically when $t$ approaches 0 [Fig. 4(b)]. In this case, by decreasing $t$, the relative contribution of the NM/FMI interface to the spin-flip scattering processes increases, enhancing $\beta$. For instance, when $t \sim 20 \mathrm{~nm}, \beta$ already increases to $\sim 50 \%$. However, the decrease of $\lambda$ with $t$ [26], which has not been taken into account for the representation, will lower $\beta$. The most effective way of improving $\beta$ seems to be increasing $G_{r}$ [Fig. 4(c)]. By increasing it by two orders of magnitude, i.e., for a $G_{r}$ of the order that $\mathrm{Pt} / \mathrm{YIG}$ systems have, $\beta$ reaches almost up to a $100 \%$, which would lead to a perfect magnetic gating of the pure spin currents. This seems feasible by improving the interface between $\mathrm{Cu}$ and YIG or by using another NM material with a high spin-mixing interface conductance with YIG.

To conclude, we present an approach to control and manipulate spins in a solid state device, by means of a magnetic gating of pure spin currents in $\mathrm{Co} / \mathrm{Cu} \mathrm{LSV}$ devices on top of YIG. A modulation of the pure spin current is observed as a function of the relative orientation between the magnetization of the FMI and the polarization of the spin current. Such modulation is explained by solving the spin-diffusion equation and considering the spin-mixing conductance at the NM/FMI interface. The accuracy between the measured data and the expected modulation provides an effective way of studying the NM/FMI interface. From our results, a spin-mixing conductance of $G_{r} \sim 4 \times 10^{11} \Omega^{-1} \mathrm{~m}^{-2}$ is obtained for the $\mathrm{Cu} / \mathrm{YIG}$ interface. An increase of this value will enhance the efficiency of the magnetic gating. This can be achieved by carefully tuning the fabrication parameters. Our experiment paves the way for different manners of spin manipulation, bringing closer pure spin currents and logic circuits.

We thank Professor Joaquín Fernández-Rossier for fruitful discussions. This work was supported by the European Commission under the Marie Curie Actions (256470ITAMOSCINOM), NMP Project (263104-HINTS) and the European Research Council (257654-SPINTROS), by the Spanish MINECO under Projects No. MAT2012-37638, No. MAT2012-36844, and No. FIS2011-28851-C02-02, and by the Basque Government under Project No. PI2012-47 and UPV/EHU Project No. IT-756-13. E.V. and M.I. thank the Basque Government for support through a Ph.D. fellowship (Grants No. BFI-2010-163 and No. BFI-2011-106). F.S.B. thanks Professor Martin Holthaus and his group for their kind hospitality at the Physics Institute of the Oldenburg University.
[1] Spin Current, edited by S. Maekawa, S. O. Valenzuela, E. Saitoh, and T. Kimura (Oxford University Press, Oxford, UK, 2012).

[2] B. Behin-Aein, D. Datta, S. Salahuddin, and S. Datta, Nat. Nanotechnol. 5, 266 (2010).

[3] S. Datta and B. Das, Appl. Phys. Lett. 56, 665 (1990).

[4] H. C. Koo, J. H. Kwon, J. Eom, J. Chang, S. H. Han, and M. Johnson, Science 325, 1515 (2009).

[5] A. Brataas, Y. V. Nazarov, and G. E. W. Bauer, Phys. Rev. Lett. 84, 2481 (2000).

[6] M. Weiler, M. Althammer, M. Schreier, J. Lotze, M. Pernpeintner, S. Meyer, H. Huebl, R. Gross, A. Kamra, J. Xiao, Y.-T. Chen, H. J. Jiao, G. E W. Bauer, and S. T. B. Goennenwein, Phys. Rev. Lett. 111, 176601 (2013).
[7] Y. Kajiwara, K. Harii, S. Takahahi, J. Ohe, K. Uchida, M. Mizuguchi, H. Umezawa, H. Kawai, K. Ando, K. Takanashi, S. Maekawa, and E. Saitoh, Nature (London) 464, 262 (2010).

[8] L. Qiu, K. Ando, K. Uchida, Y. Kajiwara, R. Takahashi, H. Nakayama, T. An, Y. Fujikawa, and E. Saitoh, Appl. Phys. Lett. 103, 092404 (2013).

[9] V. Castel, N. Vlietstra, B. J. van Wees, and J. Ben Youssef, Appl. Phys. Lett. 101, 132414 (2012).

[10] B. Heinrich, C. Burrowes, E. Montoya, B. Kardasz, E. Girt, Y. Y. Song, Y. Sun, and M. Wu, Phys. Rev. Lett. 107, 066604 (2011).

[11] C. Burrowes, B. Heinrich, B. Kardasz, E. A. Montoya, E. Girt, Y. Sun, Y.-Y. Song, and M. Wu, Appl. Phys. Lett. 100, 092403 (2012). 
[12] M. B. Jungfleisch, V. Lauer, R. Neb, A. V. Chumak, and B. Hillebrands, Appl. Phys. Lett. 103, 022411 (2013).

[13] K. Uchida, J. Xiao, H. Adachi, J. Ohe, S. Takahashi, J. Ieda, T. Ota, Y. Kajiwara, H. Umezawa, H. Kawai, G. E. W. Bauer, S. Maekawa, and E. Saitoh, Nat. Mater. 9, 894 (2010).

[14] H. Nakayama, M. Althammer, Y.-T. Chen, K. Uchida, Y. Kajiwara, D. Kikuchi, T. Ohtani, S. Geprägs, M. Opel, S. Takahashi, R. Gross, G. E. W. Bauer, S. T. B. Goennenwein, and E. Saitoh, Phys. Rev. Lett. 110, 206601 (2013).

[15] N. Vlietstra, J. Shan, V. Castel, B. J. van Wees, and J. Ben Youssef, Phys. Rev. B 87, 184421 (2013).

[16] C. Hahn, G. de Loubens, O. Klein, M. Viret, V. V. Naletov, and J. Ben Youssef, Phys. Rev. B 87, 174417 (2013).

[17] Y.-T. Chen, S. Takahashi, H. Nakayama, M. Althammer, S. T. B. Goennenwein, E. Saitoh, and G. E. W. Bauer, Phys. Rev. B 87, 144411 (2013).

[18] M. Isasa, A. Bedoya-Pinto, F. Golmar, F. Sánchez, L. E. Hueso, J. Fontcuberta, and F. Casanova, Appl. Phys. Lett. 105, 142402 (2014).

[19] S. O. Valenzuela and M. Tinkham, Nature (London) 442, 176 (2006).

[20] M. Johnson and R. H. Silsbee, Phys. Rev. Lett. 55, 1790 (1985).

[21] F. J. Jedema, M. S. Nijboer, A. T. Filip, and B. J. van Wees, Phys. Rev. B 67, 085319 (2003).

[22] Y. Ji, A. Hoffmann, J. E. Pearson, and S. D. Bader, Appl. Phys. Lett. 88, 052509 (2006).

[23] E. Villamor, M. Isasa, L. E. Hueso, and F. Casanova, Phys. Rev. B 88, 184411 (2013).

[24] S. Takahashi and S. Maekawa, Phys. Rev. B 67, 052409 (2003).

[25] G. Mihajlović, D. K. Schreiber, Y. Liu, J. E. Pearson, S. D. Bader, A. K. Petford-Long, and A. Hoffmann, Appl. Phys. Lett. 97, 112502 (2010).

[26] E. Villamor, M. Isasa, L. E. Hueso, and F. Casanova, Phys. Rev. B 87, 094417 (2013).

[27] M. Erekhinsky, A. Sharoni, F. Casanova, and I. K. Schuller, Appl. Phys. Lett. 96, 022513 (2010).

[28] F. J. Jedema, H. B. Heersche, A. T. Filip, J. J. A. Baselmans, and B. J. van Wees, Nature (London) 416, 713 (2002).

[29] X. J. Wang, H. Zou, and Y. Ji, Phys. Rev. B 81, 104409 (2010); H. Zou, S. T. Chui, X. J. Wang, and Y. Ji, ibid. 83, 094402 (2011); X. J. Wang, H. Zou, and Y. Ji, J. Appl. Phys. 105, 093907 (2009).

[30] J. C. Slonczewski, J. Magn. Magn. Mater. 159, L1 (1996); L. Berger, Phys. Rev. B 54, 9353 (1996).
[31] D. C. Ralph and M. D. Stiles, J. Magn. Magn. Mater. 320, 1190 (2008).

[32] X. Jia, K. Liu, K. Xia, and G. E. W. Bauer, Europhys. Lett. 96, 17005 (2011).

[33] S. Y. Huang, X. Fan, D. Qu, Y. P. Chen, W. G. Wang, J. Wu, T. Y. Chen, J. Q. Xiao, and C. L. Chien, Phys. Rev. Lett. 109, 107204 (2012).

[34] Y. M. Lu, J. W. Cai, S. Y. Huang, D. Qu, B. F. Miao, and C. L. Chien, Phys. Rev. B 87, 220409 (2013).

[35] A. B. Cahaya, O. A. Tretiakov, and G. E. W. Bauer, Appl. Phys. Lett. 104, 042402 (2014).

[36] The YIG was grown by liquid phase epitaxy on a (111) gadolinium gallium garnet (GGG) single crystal at Innovent e.V. (Jena, Germany).

[37] See Supplemental Material at http://link.aps.org/supplemental/ 10.1103/PhysRevB.91.020403 for experimental details, control experiments, and theory.

[38] E. Nikulina, O. Idigoras, P. Vavassori, A. Chuvilin, and A. Berger, Appl. Phys. Lett. 100, 142401 (2012).

[39] A modulation in $R_{\mathrm{NL}}$ originating from the rotation of magnetization of the Co electrodes (angles $\theta_{1}, \theta_{2}$ from the easy axis defined by the shape anisotropy) would be given by $R_{\mathrm{NL}} \propto \cos \theta$, where $\theta$ is the relative angle between Co magnetizations. The observed $\sim 8 \%$ modulation would correspond to $\theta \sim 23^{\circ}$. Since $\theta=\theta_{1}-\theta_{2}$ in the $\mathrm{P}$ case, whereas in the AP case $\theta=180^{\circ}-\theta_{1}-\theta_{2}$, this would lead to a different amplitude modulation not observed in our experiment. Only in the special case where the narrower electrode does not rotate at all $\left(\theta_{2} \sim 0\right)$ would the modulations corresponding to the $\mathrm{P}$ and AP cases be identical. The wider electrode should then rotate as much as $\theta_{1} \sim 23^{\circ}$, which has been ruled out by MOKE microscopy as described in Ref. [37].

[40] Y. Fukuma, L. Wang, H. Idzuchi, S. Takahashi, S. Maekawa, and Y. Otani, Nat. Mater. 10, 527 (2011).

[41] M. Johnson and R. H. Silsbee, Phys. Rev. B 37, 5312 (1988).

[42] F. S. Bergeret and I. V. Tokatly, Phys. Rev. B 89, 134517 (2014).

[43] M. Popinciuc, C. Józsa, P. J. Zomer, N. Tombros, A. Veligura, H. T. Jonkman, and B. J. van Wees, Phys. Rev. B 80, 214427 (2009).

[44] L. Liu, R. A. Buhrman, and D. C. Ralph, arXiv:1111.3702.

[45] J.-C. Rojas-Sánchez, N. Reyren, P. Laczkowski, W. Savero, J.-P. Attané, C. Deranlot, M. Jamet, J.-M. George, L. Vila, and H. Jaffrès, Phys. Rev. Lett. 112, 106602 (2014). 